\title{
In search of a combined brucellosis and tuberculosis vaccine for cattle
}

\author{
Gerhardt G. Schurig*, Stephen M. Boyle, \\ Hamzeh Al Qublan, Nammalwar Sriranganathan
}

\begin{abstract}
Bovine brucellosis is caused by Brucella abortus. The bacterial pathogen causes economic losses because it induces abortion in cattle. Vaccination of calves with live B. abortus strain 19 induces a certain level of protection but induces persistent antibodies against cell envelope lipopolysaccharide that make it difficult to Distinguish Infected from Vaccinated Animals (DIVA). Live vaccine $B$. abortus strain RB51 was developed to eliminate such interfering antibodies and therefore, facilitate the differentiation of infected from vaccinated animals and help in the eradication of the disease. Vaccination with strain RB51 induces levels of protection similar to strain 19 but neither of the two vaccines give complete protection. We have been working to enhance protection induced by strain RB51 vaccine. Protective Brucella antigens can be over-expressed in strain RB51 by introducing a plasmid containing the leuB gene and the genes encoding such antigens. To avoid the expression of antibiotic resistance genes, we produced a leuB deficient strain RB51 and introduced a plasmid containing the $l e u B$ gene and the genes to be over-expressed. This new strain maintains the plasmid and has induced significantly high protection levels in mice. In addition, it allowed the construction of an RB51 vaccine strain able to express Mycobacterium bovis protective antigens so that the vaccine could protect against brucellosis and tuberculosis simultaneously.

Key words: Brucella, Mycobacterium, vaccine, RB51, protection.
\end{abstract}

Animal brucellosis is a disease affecting various domestic and wild life species and is caused by an infection with bacteria belonging to the genus Brucella. The genus has several species and each species has a preference for specific animal hosts, for example, $B$. abortus mainly infects cattle, $B$. melitensis infects mainly goats and sheep and $B$. suis infects mainly pigs. These small Gram negative bacteria form a genetically coherent taxon which is related most closely to Ochrobactrum and more distantly to Agrobacterium and Rhizobium within the alpha-2 subgroup of the Proteobacteriaceae.

Most of the Brucella nomen species are transmissible to humans where it can cause serious acute and chronic disease with some cases resulting in death, making brucellosis an important zoonosis. Brucella is also a facultative intracellular parasite; the pathogenesis of brucellosis and the nature of the protective immune response are closely related to this property (Cheers 1984).

Because of the serious economic and medical consequences of brucellosis for both cattle farmers and humans in general, efforts have been made to prevent the infection through the use of vaccines (Nicoletti 1990). These were initially developed on an empirical basis, but with our current ability to manipulate the genome of the bacteria, more rational designs are being used for the development of better vaccines against the disease.

The lipopolysaccharide (LPS) molecule is closely associated with the phenotype of Brucella colonies in culture. Brucella can present itself upon culture with either a "smooth" or a "rough" colony morphology, with some

Received: 07.10.2020.

Accepted: 20.05.2020.

Virginia-Maryland College of Veterinary Medicine, Virginia Tech, Blacksburg, USA.

*Corresponding author: G Schurig; gschurig@vt.edu strains presenting a "mucoid" phenotype (White and Wilson 1951). It is possible for smooth colonies to become rough spontaneously and some rough Brucella strains may revert to the smooth morphology making the degree of stability an important consideration in vaccine development. Virulence of $B$. abortus is associated with smooth morphology and therefore, $B$. abortus field strains producing acute and chronic brucellosis in cattle (and humans) are smooth. Smooth organisms have an LPS molecules containing a polysaccharide O-chain made from a homopolymer of perosamine ( $\mathrm{N}$-formyl-4-amino,4,6-dideoxy mannose), while rough organisms lack this O-chain on their LPS molecule; some strains possess only a greatly truncated portion of it (Caroff et al 1984, Moreno 1984). The O-chain of smooth Brucella is located on the surface of the organism. Essentially all animals infected with smooth strains respond immunologically to this O-chain by making antibodies against it. This is a very important point because, all serological tests used in the diagnosis of infected cattle are based on the detection of O-chain antibodies (Diaz R et al 1968, Nielsen et al 1988, Vemulapalli et al 2000). Also, it is very important to be aware that antibodies against the $\mathrm{O}$-chain do not protect cattle against infection with $B$. abortus and in general, a clear role of any other Brucella antibodies in protection against cattle brucellosis has not been demonstrated. An effective immune response against Brucella infection in cattle requires a strong Cell Mediated Immune (CMI) response based on both, active T-helper1 and T-cytotoxic cells (Schurig et al 2002). The current understanding is that protection in cattle is highly dependent on the induction of these specific T lymphocytes and the concurrent activation of macrophages by T-helper 1 cells secreting interferon-gamma (Schurig et al 2002, Vemulapalli et al $2000^{\mathrm{c}}$ ).

The vaccine that has been most widely used to prevent bovine brucellosis is B. abortus strain 19 (Nicoletti 1990). 
This strain was first described in 1930 and was originally isolated from the milk of a Jersey cow as a virulent strain in 1923, but after being kept in the laboratory at room temperature for over a year, was found to have become attenuated (Buck 1930). This strain was able to induce a certain level of protective immunity in cattle. Effectiveness fluctuated depending on a variety of variables including age of vaccination, dose, route and prevalence of brucellosis in vaccinated herds (Nicoletti 1990). Strain 19 is an attenuated organism with a smooth morphology normally unable to grow in the presence of erythritol (Jones et al 1965). Although strain 19 is of low virulence for cattle, vaccination of pregnant cows can result in abortions. This event is rather rare, ranging from less than 1 up to $2.5 \%$ under field conditions and between 10-12\% under experimentally controlled conditions (Mingle et al 1941, Moore 1950, Manthei 1952, Beckett and Mc Diarmid 1985, Tabynov et al 2016). Intravenous injections of pregnant cattle with strain 19 induces $100 \%$ abortions, while RB51 did induce up to 25\% abortion (Palmer et al 1996). Strain 19 is pathogenic for humans and can lead to chronic infections if cases are not treated with appropriate antibiotics (Revich et al 1961, Wallach et al 2008). The most common route of human infections with strain 19 is accidental inoculation during vaccination of cattle. Strain 19 can also be found in the milk of cattle if vaccination is carried out during adulthood (Samartino et al 2000).

Since strain 19 is a smooth species of Brucella, the presence of LPS with O-chain on its surface is the reason for the appearance and persistence of O-chain antibodies in serum following administration of this vaccine (Diaz et al 1968, Nielsen et al 1988). Since these antibodies are the basis of all diagnostic tests, strain 19 vaccination prevents easy differentiation of vaccinated from infected animals and therefore, delays eradication efforts and leads to over condemnation of cattle. In order to decrease this problem to some extent, calves are vaccinated rather early in life since their antibody response to Brucella is weaker at that time. The problem becomes worse if calves are vaccinated for the first time later in life or are re-vaccinated for booster purposes since antibody levels will increase even more and be very persistent (Manthei 1952, Samartino et al 2000).

Several undesirable characteristics of strain 19, particularly its confusing effect on sero-diagnosis, led to the development of B. abortus strain RB51 vaccine (Schurig et al 1991). Strain RB51 is a highly attenuated mutant of $B$. abortus strain 2308 . It is a very stable strain meaning that it does not change colony morphology upon multiple passages in culture or animals (Schurig et al 1991, Colby 1997). Strain RB51 has a rough colony morphology and is essentially devoid of O-chain (Schurig et al 1991). The $w b o A$ gene of strain RB51, a gene involved in the biosynthesis of the O-chain, is interrupted by an IS711 element impeding the production of O-chain (Vemulapalli et al 1996, 2000 ${ }^{\mathrm{a}}$ ). Since strain RB51 lacks O-chain, vaccinated cattle do not respond to the O-chain and do not develop antibodies to this antigen. Therefore, vaccinated animals remain serologically negative in all serological diagnostic tests. Furthermore, re-vaccination at any age does not induce O-chain antibodies in the animals allowing the application of "booster" vaccinations without affecting serological diagnostic tests necessary for disease eradication (Samartino et al 2000, Dorneles et al 2015). When used in cattle, one vaccination with strain RB51 induces protection levels similar to those induced by strain 19 and protection of the vaccinated animals can vary from $65 \%$ to $100 \%$ depending on the conditions prevailing in a specific herd or experiment (Schurig et al 2002). Revaccination with strain RB51 for a second time appears to increase immunity and represents an additional advantage of RB51 over strain 19. Since 1996, millions of cattle have been immunised with strain RB51 and reversion to a virulent form has never been observed testifying to its extreme stability.

As mentioned before, CMI responses play a critical role in resistance against intracellular bacterial infections (Schurig et al 2002, Vemulapalli et al $2000^{c}$ ). It is therefore critical that vaccines are presented to the immune system in a way that they induce a $\mathrm{T}$ cell response that can protect against the infection. Live bacterial vaccines are considered essential to induce an appropriate $\mathrm{T}$ cell mediated CMI protective response. Although the exact reasons for needing live organisms to induce the right response are debatable and are probably multiple, the synthesis of antigens by the live organisms during the immune response induction phase appears critical. Replication of the vaccine strain may be less critical than synthesis of new antigens since irradiated vaccines, where the bacteria do not replicate but are able to synthesize antigens after the irradiated vaccine application, are protective. Irradiated Brucella are able to induce protection through CMI responses while killed organisms are not (Montaraz and Winter 1968, Magnani et al 2009, Moustafa et al 2011). Since live bacteria are able to induce protective CMI responses, attenuated strains of bacteria are often used as live vaccines to protect against intracellular bacterial infections. Both B. abortus strain RB51 and strain 19 are live, attenuated vaccines able to induce protection while the same vaccines rendered metabolically inactive are not unless potent adjuvants are used (Montaraz and Winter 1968). This observation has important practical implications since live, attenuated vaccines have to be handled carefully (kept cold, use only shortly after reconstituting in the field, etc) to maintain their effectiveness.

In many cases live, attenuated vaccines do not provide high levels of protection, particularly if animals are confronted with high numbers of infectious bacteria (Manthei CA 1968). For example, a Brucella vaccinated cow will have a very high likelihood of being well protected against infection if she is exposed to a low number of field $B$. abortus bacteria (Manthei CA 1968). Not only does level of exposure affects the protective ability of the vaccine, vaccine dose will also affect the protective outcome (Manthei CA 
1968, Confer et al 1985). In contrast, a cow may not be protected when exposed to the placenta and fetus of a $B$. abortus abortion where Brucella number in the hundreds of billions. Therefore, eradication of brucellosis not only depends on vaccination, it depends on a sustained vaccination program and good management where vaccinated animals are separated as much as possible from high level infection sources and where seropositive animals are consistently removed from the herd.

The fact that full protection in cattle is probably not achieved under most field conditions, made it relevant to work on approaches that could improve effectiveness of the current strain RB51 vaccine without changing its positive characteristics of being highly attenuated and leaving animals seronegative after one or multiple vaccinations. We hypothesized, some time ago, that over-expression (production of more than normal quantities) of a Brucella protective antigen by vaccine strain RB51 would result in enhancement of the vaccine's efficacy (Vemulapalli et al $2000^{\text {b }}$, Vemulapalli et al 2002). Our studies demonstrated that we could over-express $B$. abortus $\mathrm{Cu} / \mathrm{Zn}$ superoxide dismutase (SOD) protein in vaccine strain RB51(Vemulapalli et al 2002). SOD is considered to be one (He et al 2002) of probably many protective Brucella antigens and significantly increases the vaccine's protective capabilities in the murine model of brucellosis without altering the attenuation, stability or serological characteristics of the vaccine. This stimulated our interest in pursuing this approach to produce a more effective RB51 vaccine. Interestingly, even though homologous over-expression of $\mathrm{Cu} / \mathrm{Zn}$ superoxide dismutase SOD enhanced protection significantly in mice, it did not enhanced protection against $B$. abortus infection in bison and elk (Olsen et al 2009, Nol et al 2016). In bison, the overexpressing RB51-SOD vaccine was actually less efficacious and was cleared faster from the vaccinated animals than the parenteral RB51 strain (Olson et al 2009). Faster clearance may have resulted in a weaker immune response and may explain the decrease in efficacy. This indicates that the murine model is not always an indicator of what may happen in cattle or other animal species making protection experiments in cattle crucial before a new vaccine can be called more effective in the target species.

Since homologous over-expression of a protective antigen can lead to a more effective vaccine, it was logical to think that expression by strain RB51 of a protective antigen derived from an unrelated infectious agent, for which protection is mediated by a CMI response, would protect against the unrelated disease as well as against infection with Brucella. Consistent with this hypothesis, protection against infection with Neospora caninum was demonstrated in the mouse model using a strain RB51 strain expressing parasite protective antigens (Rajasekaran et al 2008). Therefore, it may be possible to create strain RB51vaccines able to protect cattle against Brucella infection as well as protect against unrelated diseases simultaneously. Our approach to create a strain RB51 vaccine able to protect against Brucella and Mycobacterium bovis infections simultaneously is described below.

In order to use strain RB51 as a platform vaccine able to induce specific immune responses against a variety of homologous and/or heterologous antigens, a plasmid containing the gene encoding the foreign antigen along with an antibiotic resistance gene (as a plasmid marker) had been employed during our developmental work carried out in the past (McQuiston et al 1995, Vemulapalli et al $2000^{c}$, Vemulapalli et al 2002). Because Brucella spp. have no naturally occurring plasmids, we adapted a broad host range plasmid that had been shown to replicate in Brucella (Kovach et al 1994). We eliminated extraneous plasmid DNA sequences and introduced a variety of promoters to allow for expression of genes derived from Brucella (homologous overexpression) as well as derived from other bacterial species (heterologous overexpression) and the resulting plasmid was designated as pNS or pLeuB (Seleem et al 2004, see figure 1).

The approach of using an antibiotic resistance gene as a selection marker has been criticized as not being environmentally safe because in the vaccinated animals, it has the potential to introduce the antibiotic resistance gene into normal and pathogenic flora. Therefore, expressing the protective antigens from a plasmid that is not dependent on an antibiotic resistance gene for selection of the desired strain, would be an advantage, as it would have a minimum environmental risk.

The leuB gene, encoding isopropyl malate dehydrogenase, is one of the four genes essential for the biosynthesis of leucine in B. abortus (Essenberg et al 1993). B. abortus is known to survive and replicate in nutrient-limited environments inside a host. Therefore, a $B$. abortus mutant lacking a gene for the biosynthesis of an essential amino acid like leucine is unlikely to survive in that environment including being able to replicate in minimal media (Essenberg et al 1993, Bacon et al 1951, Bange et al 1996). The complementation of such an auxotroph with a plasmid carrying the wild-type leuB gene (encoding the enzyme necessary for the amino acid synthesis) would provide a means of selection and maintenance of this plasmid in B. abortus under minimum media conditions. Thus, we produced a leuB mutant in strain RB51 using allelic exchange (Rajasekaran et al 2008). The resultant leuB auxotroph (RB51leuB) cannot grow in leucine-deficient conditions but when complemented with a plasmid carrying the wild-type $B$. abortus leuB gene, the leucine deficiency of RB51leuB is eliminated (Rajasekaran et al 2008). Thus, the complemented RB51leuB strain can be used to over-express homologous and/or heterologous antigens and eliminate the environmental concerns related to antibiotic resistance. Importantly, the complemented RB51leuB strain retained the basic characteristics of the original strain RB51 which are attenuation, no induction 


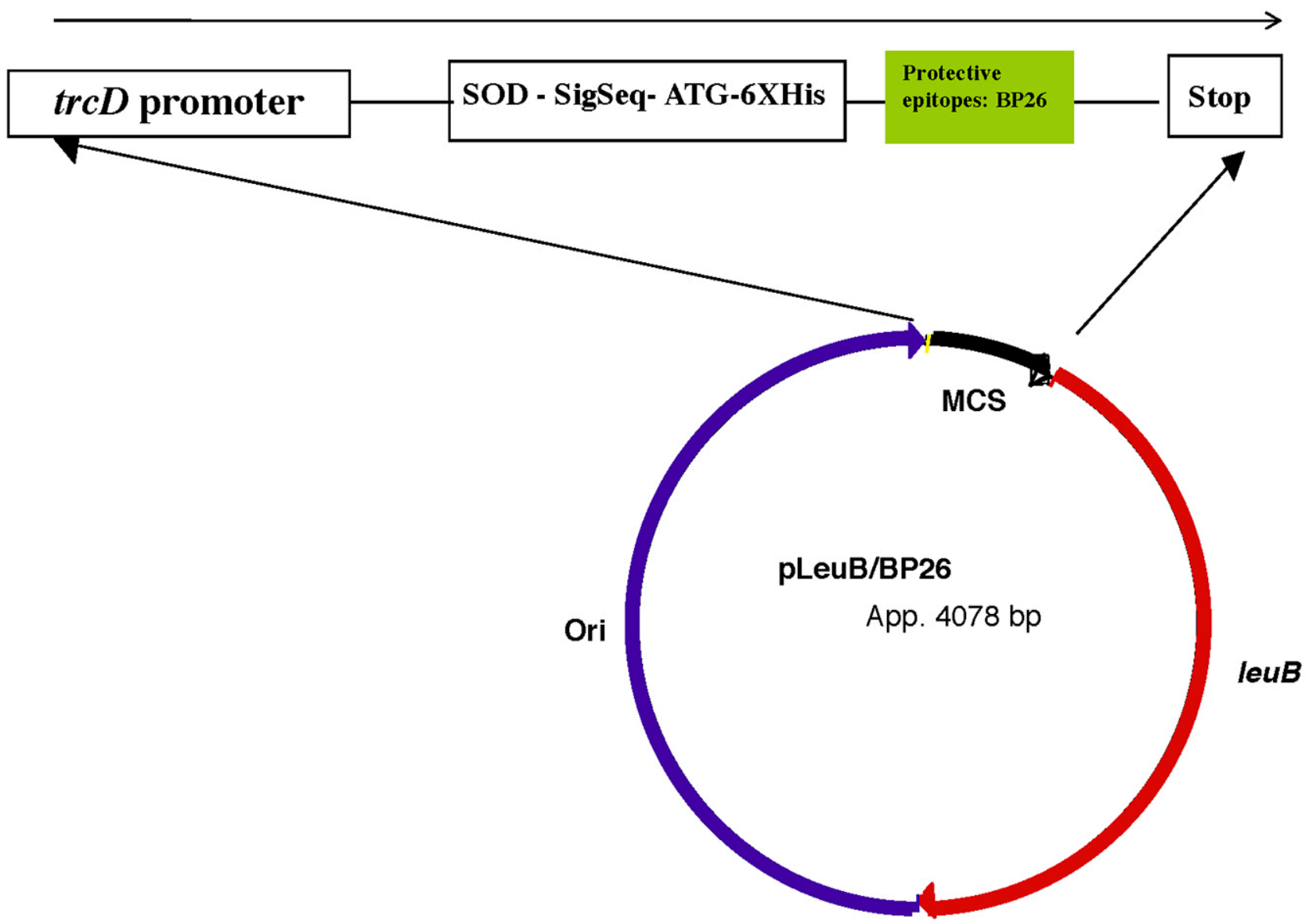

Figure 1. Schematic of pLeuB plasmid containing the leuB gene of B. abortus; a multiple cloning site (MCS) and an origin of replication (Ori). The trcD promoter is a very strong promoter as it is a hybrid between the lacZ and tryptophan promoter. The signal sequence (SigSeq) is from the SOD gene and followed by an ATG start codon and an in-frame histidine tag (6XHis) followed by a protective antigen from BP26 of B. abortus.

of O-chain antibodies and protection against $B$. abortus infection.

Using strain RB51leuB as a platform and pNSLeuB, an antibiotic-resistance marker free plasmid, we constructed several strains over-expressing homologous antigens. For example, strains RB51leuB/SOD (superoxide dismutase), $\mathrm{RB} 51$ leuB/SOD/L7/L12 (ribosomal proteins) and RB51leuB/ SOD/WboA (glycosyl transferase) were constructed to over-express the selected Brucella antigens: SOD alone, SOD and ribosomal protein L7/L12 or SOD and glycosyl transferase, respectively. The ability of these vaccine candidates to protect against a virulent $B$. suis challenge were evaluated in a mouse model. All vaccine groups protected mice significantly $(P<0.05)$ when compared to the control group. Within the vaccine groups, the mice vaccinated with strain RB51leuB/SOD/WboA were significantly better protected than those that were vaccinated with either strain $\mathrm{RB} 51$ leuB/SOD or RB51leuB/SOD/L7/L12. These results suggest that Brucella antigens can be over-expressed in strain RB51leuB and can elicit enhanced protective immune responses against brucellosis at least in the murine model of the disease (Rajasekaran et al 2011). The murine model for Brucellosis has been used by numerous authors to test protective abilities of vaccines (Montaraz and Winter 1968). Nevertheless, it is not clear if protective abilities observed in mice translate into protective abilities in cattle or other species. For example, work carried out with strain RB51 in mice indicating protection by strain RB51 was later demonstrated in cattle (Schurig et al 1991, Cheville et al 1993). On the other hand, RB51 overexpressing $\mathrm{Cu} / \mathrm{Zn}$ superoxide dismutase showed higher protection than RB51 in mice but did not protect bison against Brucella challenge (Vemulapalli et al 2002, Olson et al 2009). It remains to be seen if similar results can be obtained in cattle.

Control of bovine tuberculosis is an important element in the control or eradication of human tuberculosis. Unfortunately, there is no practical vaccine available for the control of tuberculosis in cattle even so, the problem is very significant. To illustrate the dimension of the problem, long term data analysis of bovine tuberculosis in India suggests that approximately $7.3 \%$ of cattle are potentially infected. This means that there are 21.8 million 
TB infected cattle, which amounts to more than all cattle in United States. This systematic analysis is even more alarming as approximately $10 \%$ of human tuberculosis is due to $M$. bovis infection. Therefore, control of bovine tuberculosis is an important component related to efforts of human tuberculosis eradication, especially in the developing world, as test and slaughter may not be an option due to economic and religious considerations (Srinivasan et al 2018).

Bacille Calmette Guerin (BCG) vaccine is the current TB vaccine used in humans. It is an attenuated strain of M. bovis (Behr et al 1997, Murray 2004). BCG was attenuated by serially passing a virulent $M$. bovis strain on ox-bile medium for 230 times in the laboratory. This process led to attenuation of the virulent $M$. bovis strain as indicated by self-limiting infection as well as partial resistance to reinfection with $M$. bovis (Takamura et al 2005). One of the serious disadvantages of BCG vaccine is that vaccinated humans and animals turn skin test positive. Therefore, BCG is rarely used in cattle to protect against bovine tuberculosis (Waters et al 2012). Recent, largescale field trials with cattle using very low doses of BCG demonstrated high levels of protection (vaccine efficacy between $85.7 \%-86.7 \%$ ) without affecting their ante mortem skin or blood tests (Nugent et al 2018). This low dose approach may make vaccination of cattle with BCG more acceptable than in the past.

Following our previously defined strategy of over-expressing heterologous antigens in strain RB51, we speculated that expression of protective antigens from M. bovis in $B$. abortus strain RB51, a vaccine strain that is USDA approved and extensively used throughout the world, could make an effective dual vaccine able to protect against Brucellosis and Tuberculosis in cattle simultaneously. To prove this point, we selected the following protective Mycobacterium antigens: 85B, ESAT6 and Rv2660c for this purpose (Al Qublan, Dissertation, Virginia Tech, 2014). These genes were cloned and expressed in our auxotrophic leuB deletion mutant of strain RB51 (RB51leuB). As explained before, the advantage of using the RB51leuB deletion mutant is that one can have a plasmid encoding the protective antigens along with the $l e u B$ gene instead of a drug resistance gene for selection purposes

The reasons for selecting these antigens are outlined by characteristic that define them as protective antigens.

\section{ANTIGEN 85B}

Antigen $85 \mathrm{~B}(\mathrm{Ag} 85 \mathrm{~B})$ is one of the most dominant protein antigens secreted by all mycobacterial species (Belisle et al 1997). Ag85B (30 kDa) belongs to the Ag85 complex, which is a family of three structurally related fibronectin-binding proteins ( $\mathrm{Ag} 85 \mathrm{~A}, \mathrm{Ag} 85 \mathrm{~B}$ and $\mathrm{Ag} 85 \mathrm{C})$ with mycolyl-transferase activity that is involved in the final stages of cell wall assembly. Ag85B protein is not only the major secretory protein of M. tuberculosis in broth culture, but it is also among the major proteins of all M. bovis proteins expressed and, it is a major stimulator of T-cell proliferation and IFN- $\gamma$ production in most healthy-looking animals infected with $M$. bovis. Studies have shown that immunisation with plasmid DNA encoding Ag85B can stimulate strong cell-mediated immune response and confer significant protection to mice challenged with virulent Mycobacterium (Lozes et al 1997). All these findings suggest that $\mathrm{Ag} 85 \mathrm{~B}$ is a promising protective antigen for vaccine use in other animal species.

\section{EARLY SECRETORY ANTIGENIC TARGET 6}

Early secretory antigenic target 6 (ESAT6) is a protein encoded by the region of difference 1 (RD1) of the $M$. bovis genome. RD1 has been shown to be a major virulence factor involved with membrane-lysing activity (Smith et al 2008, Gao et al 2004). Although the exact function of ESAT6 has not been determined, studies have shown that deletion of the ESAT6 protein results in abrogation of the necrosis-inducing effect of tuberculosis on human monocyte-derived macrophages; suggesting that ESAT6 is involved with causing necrosis (Welin et al 2011). ESAT6 is a strongly recognized T-cell antigen in the first phase of infection and has demonstrated protective efficacy as a subunit vaccine in animal models (Brant et al 2000), a DNA vaccine (Qingtao et al 2013), and a recombinant BCG vaccine (Pym et al 2003).

\section{RV2660C}

Rv2660c is a newly recognised antigen of unknown function that was first reported in a gene expression profiling study by Betts and colleagues (Betts et al 2003). In their study, it was reported that in nutrient-starved cultures, expression of Rv2660c increased 100 to 300 fold making it the most strongly up-regulated of all nutrient starvation-induced genes identified. In another gene expression profiling study in a mouse model, it was found that Rv2660c was expressed at high levels during early and late stages of infection (Aagaard et al 2011). More importantly, Govender and colleagues reported that Rv2660c was preferentially recognized by patients with latent TB compared with those with active tuberculosis (Govender et al 2010). These findings suggest that Rv2660c is involved in latency and therefore provides a promising vaccine candidate for targeting tuberculosis infection as it transitions into latency.

Preliminary studies carried out in our laboratories, indicated that it was crucial that the DNA sequences of the protective Mycobacterium antigens to be cloned into our plasmid were synthesised using the codon usage of Brucella. The original ESAT6 sequence had a low codon optimization Index (COI) of 0.47 in Brucella and when optimized for strain RB51 expression, the COI changed to 0.95 . Similarly, the Ag85b COI changed from 0.51 to 
0.94 and Rv2660C COI changed from 0.29 to 0.93 . These optimised sequences were used in the generation of the synthetic genes encoding the three protective antigens (Genscript NJ, USA). BamHI, BglII and XbaI sites were engineered into the sequences and the synthetic genes were then cloned into pLeub plasmid. Two strains were constructed one expressing 85B (RB51leuB/85B) and one expressing Rv2660c and ESAT6 as a fusion protein (RB51leuB/Rv2660c-ESAT6). The strain constructs were verified by PCR using specific primers for directional cloning.

After confirmation of expression of the tuberculosis protective antigens by Western blot, the above two vaccine constructs, RB51leuB/85B and RB51leuB/Rv2660cESAT6 were mixed in equal proportions and the vaccine denominated RB51TB and it was tested for its protective ability in one group of $\mathrm{BALB} / \mathrm{c}$ mice. $\mathrm{BCG}$ immunisation testing was carried out in a second group of mice and PBS was used as the non-immunised control. The mice were vaccinated intraperitoneally (ip), boost vaccinated through the same route 4 weeks later and then challenged ip with M. tuberculosis four weeks after the booster vaccination. Four weeks after challenge, mice were killed and lungs and spleen were cultured to determine Colony Forming Units (CFUs) per organ. Vaccinated mice had significantly less CFUs in each cultured organ when compared to the non-vaccinated control group indicating good protection. There was no significant protection difference among the mice vaccinated with BCG and our strain RB51 vaccine constructs. These results clearly suggest that heterologous expression of protective tuberculosis antigens in strain RB51 can lead to protection against infection with Mycobacterium at least in the murine model. Mice were also protected against challenge with $B$. abortus indicating again that expression of heterologous antigens by strain RB51leuB does not alter its protective effect against brucellosis.

If the protective ability against mycobacterial infection in the murine model can be replicated in cattle, strain RB51TB vaccine could be used to protect cattle against brucellosis and tuberculosis simultaneously. In order for this vaccine to have practical application in the field, it not only has to protect against the two diseases, it should not induce positive tuberculin skin reactions in the vaccinated animals. If positive tuberculin reactions would be induced, the practical use of the vaccine would be limited.

In order to assess if RB51TB would induce positive tuberculin reactions after vaccination, eight tuberculin negative calves were immunised with RB51TB and booster vaccinated 4 months after the first vaccine application. A tuberculin test was carried out 4 months after the booster (unpublished). All cattle remained tuberculin negative suggesting that vaccination of cattle with RB51TB will not introduce problems related to tuberculosis and brucellosis diagnosis. One major challenge still remains, that is, to demonstrate that RB51TB protects cattle against tuberculosis. Such protection experiments, including safety and stability experiments, will be very costly and time consuming but will be necessary before approval to use this promising vaccine is granted.

\section{REFERENCES}

Aagaard C, Hoang T, Dietrich J, Cardona PJ, Izzo A, et al. 2011. A multistage tuberculosis vaccine that confers efficient protection before and after exposure. Nat Med 17, 189-194.

Bacon GA, Burrows, TW, Yates M. 1951. The effects of biochemical mutation on the virulence of Bacterium typhosum; the loss of virulence of certain mutants. Br J Exp Pathol 32, 85-96.

Bange FC, Brown AM, Jacobs WR Jr. 1996. Leucine auxotrophy restricts growth of Mycobacterium bovis BCG in macrophages. Infect Immun 64, 1794-1799.

Beckett FW, Mc Diarmid SC. 1985. The effect of reduced-dose Brucella abortus strain 19 vaccination in accredited dairy herds. Br Vet J141, 507.

Behr MA, Small PM. 1997. Has BCG attenuated to impotence? Nature 389, 133-134

Belisle JT, Vissa VD, Sievert T, Takayama K, Brennan PJ, et al. 1997. Role of the major antigen of Mycobacterium tuberculosis in cell wall biogenesis. Science 276, 1420-1422.

Betts JC, Lukey PT, Robb LC, McAdam RA, Duncan K. 2002. Evaluation of a nutrient starvation model of Mycobacterium tuberculosis persistence by gene and protein expression profiling. Mol Microbiol 43, 717-731.

Brandt L, Elhay M, Rosenkrands I, Lindblad EB, Andersen P. 2000. ESAT-6 subunit vaccination against Mycobacterium tuberculosis. Infect Immun 68, 791-795.

Buck JM. 1930. Studies of vaccination during calfhood to prevent bovine infectious abortion. J Agric Res 41, 667-689.

Caroff M, Bundle, DR, Perry MB, Cherwonogrodzky JW, Duncan JR. 1984. Antigenic S-type lipopolysaccharide of Brucella abortus 1119-3. Infect Immun 46, 384.

Cheers C. 1984. Pathogenesis and cellular immunity in experimental murine brucellosis. Dev Biol Stand 56, 237-246.

Cheville NF,Stevens MG,Jensen AE,Tatum FM,Halling SM. 1993. Immune responses and protection against infection and abortion in cattle experimentally vaccinated with mutant strains of Brucella abortus. Am J Vet Res 54, 1591-1597.

Colby LA. 1997. The humoral response of Elk (Cervus elaphus nelsoni) and mice to vaccination with Brucella abortus strain RB51. M.Sc. thesis, Virginia Polytechnic Institute and State University, Blacksburg, VA, USA.

Confer AW, Hall SM, Faulkner CB, Espe BH, Deyoe BL, et al. 1985. Effects of challenge dose on the clinical and immune responses of cattle vaccinated with reduced doses of Brucella abortus strain 19. Vet Microbiol 10, 561-575.

Crawford RP, Adams LG, Ficht TA, Templeton JW, Williams JD. 1991. Effect of stage of gestation on efficacy of Brucella abortus strain-19 vaccination in cattle. Am J Vet Res 52, 1848-1851.

Diaz R, Jones LM, Leong D, Wilson JB. 1968. Surface antigens of smooth brucellae. J Bacteriol 96, 893-901.

Dorneles EM, Lima GK, Teixeira-Carvalho A, Araújo MS, MartinsFilho OA, et al. 2015. Immune response of calves vaccinated with Brucella abortus S19 or RB51 and revaccinated with RB51. PLoS One 10, e0136696.

Essenberg R C, Sharma YK. 1993. Cloning of genes for proline and leucine biosynthesis from Brucella abortus by functional complementation in Escherichia coli. J Gen Microbiol 139, 87-93.

Gao LY, Guo S, McLaughlin B, Morisaki H, Engel JN, et al. 2004. A mycobacterial virulence gene cluster extending RD1 is required for cytolysis, bacterial spreading and ESAT-6 secretion. Mol Microbiol 53, 1677-1693.

Govender L, Abel B, Hughes EJ, Scriba TJ, Kagina BM, et al. 2010. Higher human CD4 T cell response to novel Mycobacterium tuberculosis 
latency associated antigens Rv2660 and Rv2659 in latent infection compared with tuberculosis disease. Vaccine 29, 51-57.

He Y, Vemulapalli R, Zeytun A, Schurig GG. 2001 Induction of specific cytotoxic lymphocytes in mice vaccinated with Brucella abortus RB51. Infect Immun 69,5502-5508.

He Y, Vemulapalli R, Schurig GG. 2002. Recombinant Ochrobactrum anthropi expressing Brucella abortus $\mathrm{Cu}, \mathrm{Zn}$ superoxide dismutase protects mice against $B$. abortus infection only after switching of immune responses to Th1 type. Infect Immun 70, 2535-2543.

Jiang Q, Zhang J, Chen X, Xia M, Lu Y, et al. 2013. A novel recombinant DNA vaccine encoding Mycobacterium tuberculosis ESAT-6 and FL protects against Mycobacterium tuberculosis challenge in mice. J Biomed Res 27, 406-420.

Jones LM, Montgomery V, Wilson JB. 1965. Characteristics of carbon dioxide independent cultures of Brucella abortus isolated from cattle vaccinated with strain 19. J Infect Dis 115, 312-320.

Kovach ME, Phillips RW, Elzer PH, Roop RM, 2nd, Peterson KM. 1994. pBBR1MCS: a broad host-range cloning vector. Biotechniques 16, 800-802.

Lozes E, Huygen K, Content J, Denis O, Montgomery DL, et al. 1997. Immunogenicity and efficacy of a tuberculosis DNA vaccine encoding the components of the secreted antigen 85 complex. Vaccine $15,830-833$.

McQuiston JR, Schurig GG, Sriranganathan N, Boyle SM. 1995. Transformation of Brucella species with suicide and broad hostrange plasmids. Methods Mol Biol 47,143-148.

Magnani DM, Harms JS, Durward MA, Splitter GA. 2009. Nondividing but metabolically active gamma-irradiated Brucella melitensis is protective against virulent $B$. melitensis challenge in mice. Infect Immun 77, 5181-5189.

Manthei CA. 1952. Evaluation of vaccinal methods and doses of Brucella abortus strain 19. In: Proceedings of the $56^{\text {th }}$ Annual Meeting of Livestock Sanitation Association, 115-125.

Manthei CA. 1968. Brucellosis. Application of research to bovine brucellosis control and eradication programs. J Dairy Sci 51,1115-1120.

Mingle CK, Manthei CA, Jasmin AM. 1941. The stability of reduced virulence exhibited by Brucella abortus. J Am Vet Med Asso 99, 203.

Montaraz JA, Winter AJ. 1986. Comparison of living and nonliving vaccines for Brucella abortus in BALB/c mice. Infect Immun 53, 245-251.

Moore T, Mitchell CA. 1950. Vaccination of sexually mature cows with Brucella abortus strain 19 vaccine. Can J Comp Med Vet Sci 14, 209-213.

Moreno E. 1984. Immunochemical characterization of rough Brucella lipopolysaccharides. Infect Immun 43, 779.

Moustafa D, Garg VK, Jain N, Sriranganathan N, Vemulapalli R. 2011. Immunization of mice with gamma-irradiated Brucella neotomae and its recombinant strains induces protection against virulent $B$. abortus, B. melitensis, and B. suis challenge. Vaccine 29, 784-794.

Murray JF. 2004. A century of tuberculosis. Am J Resp Cr Care Med 169, 1181-1186.

Nicoletti P. 1990. Vaccination. In: Nielsen K, Duncan JR (eds). Animal Brucellosis. CRC Press, Boca Raton, FL, USA, Pp 284-299.

Nielsen KH, Wright PF, Kelly WA, Cherwonogrodzky JH. 1988. A review of enzyme immunoassay for detection of antibody to Brucella abortus in cattle. Vet Immunol Immunopathol 18, 331-347.

Nol P, Olsen SC, Rhyan JC, Sriranganathan N, McCollum MP, et al. 2016. Vaccination of Elk (Cervus canadensis) with Brucella abortus strain RB51 over-expressing superoxide dismutase and glycosyltransferase genes does not induce adequate protection against experimental Brucella abortus challenge. Front Cell Infect Microbiol 6, 10.

Nugent G, Yockney IJ, Cross ML, Buddle BM. 2018. Low-dose BCG vaccination protects free-ranging cattle against naturally-acquired bovine tuberculosis. Vaccine 36, 7338-7344.

Olsen SC, Boyle SM, Schurig GG, Sriranganathan N. 2009. Immune responses and protection against experimental challenge after vaccination of bison with Brucella abortus strain RB51 or RB51 overexpressing superoxide dismutase and glycosyltransferase genes. Clin Vaccine Immunol 16, 535-540.

Palmer MV, Cheville NF, Jensen AE. 1996. Experimental infection of pregnant cattle with the vaccine candidate Brucella abortus strain RB51: pathologic, bacteriologic, and serologic findings. Vet Pathol 33, 682-691.

Pym AS, Brodin P, Majlessi L, Brosch R, Demangel C, et al. 2003. Recombinant BCG exporting ESAT-6 confers enhanced protection against tuberculosis. Nat Med 9, 533-539.

Rajasekaran P, Seleem MN, Contreras A, Purwantini E, Schurig GG, et al. 2008. Brucella abortus strain RB51 leucine auxotroph as an environmentally safe vaccine for plasmid maintenance and antigen over-expression. Appl Environ Microbiol 74, 7051-7055.

Rajasekaran P, Surendran N, Seleem MN, Sriranganathan N, Schurig GG, et al. 2011. Over-expression of homologous antigens in a leucine auxotroph of Brucella abortus strain RB51 protects mice against a virulent $B$. suis challenge. Vaccine 29, 3106-3110.

Revich SJ, Walker AW, Pivnick H. 1961. Human infection by Brucella abortus strain 19. Can J Public Health 52, 285-289.

Samartino LE, Fort M, Gregoret R, Schurig GG. 2000. Use of Brucella abortus vaccine strain RB51 in pregnant cows after calfhood vaccination with strain 19 in Argentina. Prev Vet Med 45, 193-199.

Seleem MN, Vemulapalli R, Boyle SM, Schurig GG, Srirangnathan N. 2004. Improved expression vector for Brucella species. Biotechniques 37, 740-744.

Seleem MN, Jain N, Alqublan H, Vemulapalli R, Boyle S, et al. 2008. Activity of native vs. synthetic promoters in Brucella. FEMS Microbiology Letters 288, 211-215.

Schurig GG, Roop RM, Bagchi T, Boyle S, Buhrman D, Srirangananathan N. 1991. Biological properties of RB51; a stable rough strain of Brucella abortus. Vet Microbiol 28, 171-188.

Schurig GG, Sriranganathan N, Corbel MJ. 2002. Brucellosis vaccines: Past, present and future. Vet Microbiol 90, 479-496.

Smith J, Manoranjan J, Pan M, Bohsali A, Xu J, et al. 2008. Evidence for pore formation in host cell membranes by ESX-1-secreted ESAT-6 and its role in Mycobacterium marinum escape from the vacuole. Infect Immun 76, 5478-5487.

Srinivasan S, Easterling L, Rimal B, Niu XM, Conlan AJK, et al. 2018. Prevalence of bovine tuberculosis in India: A systematic review and meta-analysis. Transbound Emerg Dis 65, 1627-1640.

Tabynov K, Orynbayev M, Renukaradhya GJ, Sansyzbay A. 2016. Simultaneous subcutaneous and conjunctival administration of the influenza viral vector-based Brucella abortus vaccine to pregnant heifers provides better protection against $B$. abortus 544 infection than the commercial B. abortus $\mathrm{S} 19$ vaccine. Vaccine 34, 5049-5052.

Takamura S, Matsuo K, Takebe Y, Yasutomi Y. 2005. Ag85B of mycobacteria elicits Effective CTL responses through activation of robust Th1 immunity as a novel adjuvant in DNA vaccine. $J$ Immunol $175,2541-2547$

Vemulapalli R, McQuiston JR, Schurig GG, Sriranganathan N, Halling, et al. 1996. Identification of an IS711 element interrupting the wboA gene of Brucella abortus strain RB51 and a PCR assay to distinguish strain RB51 from other Brucella species and strains. Clin Diagn Lab Immunol 6, 760-764.

Vemulapalli R, He Y, Buccolo LS, Boyle S, Sriranganathan N, et al. 2000. Complementation of Brucella abortus RB51 with a functional wboA gene results in $\mathrm{O}$-antigen synthesis and enhanced vaccine efficacy but no change in rough phenotype and attenuation. Infect Immun 68, 3927-3932.

Vemulapalli R, He Y, Buccolo LS, Boyle S, Sriranganathan N, et al. $2000^{a}$. Complementation of Brucella abortus RB51 with a functional wboA gene results in $\mathrm{O}$-antigen synthesis and enhanced vaccine efficacy but no change in rough phenotype and attenuation. Infect Immun 68, 3927-3932.

Vemulapalli R, He Y, Cravero S, Sriranganathan N, Boyle S, et al. $2000^{\mathrm{b}}$. Over-expression of protective antigen as a novel approach 
to enhance vaccine efficacy of Brucella abortus strain RB51. Infect Immun 68, 3286-3289.

Vemulapalli R, He Y, Boyle S, Sriranganathan N, Schurig GG. 2000 Brucella abortus strain RB51 as a vector for heterologous protein expression and induction of specific Th1 type immune responses. Infect Immun 68, 3290-3296.

Vemulapalli R, He Y, Sriranganathan N, Boyle S, Schurig GG. 2002 Brucella abortus RB51: enhancing vaccine efficacy and developing multivalent vaccines. Vet Microbiol 90, 521-532.

Wallach JC, Ferrero MC, Delpino MV, Fossati CA, Baldi PC. 2008. Occupational infection due to Brucella abortus S19 among workers involved in vaccine production in Argentina. Clin Microbiol Infect 14, 805-807.

Waters WR, Palmer MV, Buddle BM, Vordermeier HM. 2012. Bovine tuberculosis vaccine research: historical perspectives and recent advances. Vaccine 30, 2611-2622.

Welin A, Eklund D, Stendahl O, Lerm M. 2011. Human macrophages infected with a high burden of ESAT-6-expressing M. tuberculosis undergo caspase-1- and cathepsin B-independent necrosis. PLoS ONE 6, e20302.

White PG, Wilson JB. 1951. The differentiation of smooth and nonsmooth colonies of Brucellae. J Bacteriol 61, 239-240. 